Bull. Korean Math. Soc. 49 (2012), No. 2, pp. 373-379

http://dx.doi.org/10.4134/BKMS.2012.49.2.373

\title{
SOME HOMOGENEITY CLASSES OF POSETS OF HEIGHT 2
}

\author{
Gab-Byung Chae, Minseok Cheong, and Sang-Mok Kim
}

\begin{abstract}
In this paper, we find the inclusion relation among four categories of posets, i.e., ideal-homogeneous, tower-homogeneous, quasi-complement-preserved, and complement-preserved posets.
\end{abstract}

\section{Introduction}

It is a well-known problem to give classifications of those which satisfy certain homogeneity conditions in graph theory [3], and a characterization of countable partially ordered sets was given in [5]. It is very natural to ask whether every isomorphism between finite substructures can be extendable to an automorphism of the whole structure. A few decades later, some results on the homogeneity for finite partially ordered sets are also given by G. Behrendt [1], and they make resume to investigate the relationship between the homogeneity conditions for finite partially ordered sets.

Suppose $(P, \leqslant)$ is a finite partially ordered set (simply called a finite poset) with a partial order relation $\leqslant$, which is simply denoted by $P$ for convenience. If $Q \subset P$, we may refer to $Q$ also as a poset, having in mind the subposet $(Q, \leqslant)$ whose order relation is the restriction of $(P, \leqslant)$ 's.

A chain is said to be maximal if it is not a proper subposet of any other chain. A maximum chain is a maximal chain with the maximum cardinality. The height of a poset $P$, denoted by $h t(P)$, is the number of points in a maximum chain, and the length is one less than the height, denoted by $l(P)$. An element $x \in P$ is maximal if there is no element $y(\neq x) \in P$ such that $x \leqslant y$. For an element $x \in P$, the height $h t(x)$ is the maximal cardinality of chains in $\{y \in P \mid y \leqslant x\}$. For a positive integer $n$, let $H_{n}(P, \leqslant)=\{x \in P \mid h t(x)=n\}$.

We say that $x$ is covered by $y$ in $P$ (also, $y$ covers $x$ in $P$ and $(x, y)$ is a covering pair in $P$ ) when $x \leqslant y$ and there is no $z \in P$ with $x \neq z \neq y$ such that $x \leqslant z \leqslant y$. For a covering pair $(x, y), y$ is called a up-cover of $x$, and $x$ is called a down-cover of $y$. Let $U \operatorname{Cov}(x)$ be the set of all up-covers of $x$. Define

Received November 8, 2010; Revised August 5, 2011.

2010 Mathematics Subject Classification. 06A07.

Key words and phrases. ideal-homogeneous, tower-homogeneous, quasi-complementpreserved poset, complement-preserved poset.

The first author was supported by Wonkwang University in 2011.

(C)2012 The Korean Mathematical Society 
updeg $(x)$ as $|U \operatorname{Cov}(x)|$. Similarly, let $D \operatorname{Cov}(x)$ be the set of all down-covers of $x$, and downdeg $(x)=|D C \operatorname{Cov}(x)|$.

For a poset $P$ and $x \in P$, let $U[x]=\{y \in P \mid y \geqslant x$ in $P\}$ and $D[x]=$ $\{y \in P \mid y \leqslant x$ in $P\}$. Also, we let $U[A]=\cup_{x \in A} U[x]$ and $D[A]=\cup_{x \in A} D[x]$ for a subposet $A$ of $P$. Let $U(x)=\{y \in P \mid y \geqslant x$ in $P\} \backslash\{x\}$ and $D(x)=$ $\{y \in P \mid y \leqslant x$ in $P\} \backslash\{x\}$. Also, we let $U(A)=\cup_{x \in A} U(x) \backslash A$ and $D(A)=$ $\cup_{x \in A} D(x) \backslash A$ for a subposet $A$ of $P$.

The dual of a poset $P$, denoted by $P^{d}=(P, \leqslant)^{d}$, is defined as $\left(P, \leqslant{ }^{d}\right)$ where $x \leqslant y$ in $P$ if and only if $y \leqslant{ }^{d} x$ in $P^{d}$. A poset $P$ is self-dual if $P$ is isomorphic to $P^{d}$.

A map $f:(P, \leqslant) \rightarrow\left(Q, \leqslant^{\prime}\right)$ of posets is order-preserving if $x \leqslant y$ implies $f(x) \leqslant f(y)$ for all $x, y \in P$. If $x \leqslant y$ implies $f(y) \leqslant f(x)$, the map is order-reversing. Two posets $(P, \leqslant)$ and $\left(Q, \leqslant^{\prime}\right)$ are isomorphic if there exists an order-preserving bijection $f:(P, \leqslant) \rightarrow\left(Q, \leqslant^{\prime}\right)$ such that $f^{-1}$ is also orderpreserving. A bijection $f:(P, \leqslant) \rightarrow(P, \leqslant)$ is an automorphism if $f$ and $f^{-1}$ are order-preserving, and an antiautomorphism if $f$ and $f^{-1}$ are order-reversing. We denote the set of all automorphisms of a poset $P$ by $\operatorname{Aut}(P)$.

An ideal $I$ of $P$ is a non-empty subset of $P$ such that if $x \leqslant y$ for $x \in P$ and $y \in I$, then $x \in I$. A poset $P$ is ideal-homogeneous, provided that, for any ideals $I$ and $J$ with $I \cong{ }_{\sigma} J$, there exists an automorphism $\sigma^{*} \in$ Aut $P$ such that $\left.\sigma^{*}\right|_{I}=\sigma$. A poset $P$ is weakly ideal-homogeneous, provided that for each $I$ of $P$ and $\sigma \in \operatorname{Aut}(I)$, there is $\sigma^{*} \in \operatorname{Aut}(P)$ such that $\left.\sigma^{*}\right|_{I}=\sigma$.

A subset $S$ of $P$ is called a tower in $P$ if for every $x \in S$ there exists a maximal chain $C$ in $\{y \in P \mid y \leqslant x\}$ such that $C \subset S$. We call a poset $P$ towerhomogeneous if for two isomorphic towers $S_{1}$ and $S_{2}$ with an isomorphism $\sigma: S_{1} \rightarrow S_{2}$, there exists an automorphism $\beta$ in $\operatorname{Aut}(P)$ such that $\sigma(x)=\beta(x)$ for all $x \in S_{1}$. We say that $(P, \leqslant)$ is weakly tower-homogeneous if for each tower $S$ and each automorphism $\sigma \in \operatorname{Aut}(S)$, there exists an automorphism $\beta$ in $\operatorname{Aut}(P)$ such that $\sigma(x)=\beta(x)$ for all $x \in S$. Throughout this paper, let $\mathbb{N}$, $\mathbb{Z}$, and $[n]$ denote the set of all natural numbers, the set of all integers, and $\{x \in \mathbb{Z} \mid 1 \leq x \leq n\}$, respectively.

The following two theorems, due to Behrendt [1], characterizes the (weakly) ideal-homogeneous posets and (weakly) tower-homogeneous posets of height 2 , respectively.

Theorem $1.1([1])$. Let $(P, \leqslant)$ be a finite partially ordered set of height-two. The followings are equivalent.

(i) $(P, \leqslant)$ is ideal-homogeneous.

(ii) $(P, \leqslant)$ is weakly ideal-homogeneous.

(iii) There exist a positive integer $n$ and a function $f:[n] \rightarrow \mathbb{N}$ such that there exists $i \in[n]$ with $f(i) \neq 0$ and $(P, \leqslant)$ is isomorphic to $(X, \leqslant)$ where

$$
X=[n] \cup\{(S, i) \mid \emptyset \neq S \subseteq[n], 1 \leq i \leq f(|S|)\}
$$




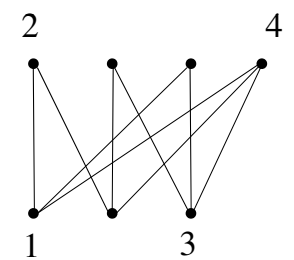

$P$

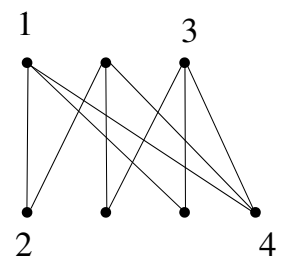

$P^{d}$

Figure 1. P is QCPP and ideal-homogeneous poset which is neither tower-homogeneous nor CPP

$$
\begin{array}{r}
\text { and for } k \in[n], \emptyset \neq S \subseteq[n], 1 \leq i \leq f(|S|) \text {, let } \\
k \leqslant(S, i) \quad \text { if and only if } k \in S .
\end{array}
$$

For integers $p, q \geq 1$, let $C(p, q)$ be the linear sum $R_{1} \oplus R_{2}$ of two disjoint antichains $R_{1}=\left\{a_{1}, \ldots, a_{p}\right\}$ and $R_{2}=\left\{b_{1}, \ldots, b_{q}\right\}$, i.e., all $a_{i}$ 's in $R_{1}$ are incomparable, and all $b_{j}$ 's in $R_{2}$ are also incomparable, and $a_{i} \leqslant b_{j}$ for all $a_{i} \in$ $R_{1}$ and $b_{j} \in R_{2}$. For integers $n, k>1$, let $A(n, k)$ be the disjoint sum of $n$ copies of $C(1, k)$. For $n \geq 3$ let $B(n)$ be the poset consisting of 1-element subsets and $(n-1)$-element subsets of $\{1, \ldots, n\}$, ordered by set-theoretic inclusion.

Theorem $1.2([1])$. Let $(P, \leqslant)$ be a finite height-two ordered set. The following are equivalent.

(i) $(P, \leqslant)$ is tower-homogeneous.

(ii) $(P, \leqslant)$ is weakly tower-homogeneous.

(iii) $(P, \leqslant)$ is isomorphic to $C(p, q)$ for some $p, q \geq 1$, or to $A(n, k)$ for some $n, k \geq 1$ or to $B(n)$ for $n \geq 3$.

Definition 1 ([4]). Let $I$ and $J$ be ideals of a poset $P$. Then $P$ is called a quasi-complement-preserved poset $(Q C P P)$ if $I \cong J$ in $P$, then $I^{c} \cong J^{c}$ in $P^{d}$.

It looks like that, for two isomorphic ideals $I$ and $J$ of $P^{d}$, if $P$ is a QCPP, then $I^{c} \cong J^{c}$ in $P$. However, in Figure $1, I=\{2\}$ and $J=\{4\}$ are isomorphic in $P^{d}$, but $I^{c}$ and $J^{c}$ are not isomorphic in $P$ while $P$ is a QCPP. Hence we can define a type of poset which is a QCPP satisfying the converse of Definition 1, as follows.

Definition 2. A poset $P$ is a complement-preserved poset $(C P P)$ if $P$ and $P^{d}$ are QCPPs.

In this paper, we find the relationship among these four homogeneity classes of posets of height 2 . 


\section{Ideals in a quasi-complement-preserved poset}

Lemma 2.1. For a poset $P$, if $I$ is an ideal in $P$, then $I^{c}$ is an ideal in $P^{d}$.

Proof. Let $x \in I^{c}$ and $y \leqslant x$ in $P^{d}$. Then $x \leqslant y$ in $P$. Since $x \in I^{c}$, we have $x \notin I$. Since $x \leqslant y$ in $P$, we have $y \notin I$, i.e., $y \in I^{c}$. Therefore $I^{c}$ is an ideal in $P^{d}$.

Lemma 2.2. If $P$ is a $C P P$ and $x, y \in H_{1}(P, \leqslant)$, then updeg $x=\operatorname{updeg} y$.

Proof. Suppose $I=\{x\}$ and $J=\{y\}$ for any elements $x, y$ in $H_{1}(P, \leqslant)$. Then $I \cong J$. Since $P$ is a CPP, we have $I^{c} \cong J^{c}$. Let $m$ be the number of edges in $P$ when, temporarily, we regard $P$ as a graph, and $m^{\prime}$ be the number of edges in $I^{c}$ and $m^{\prime \prime}$ be the number of edges in $J^{c}$. Since $I=\{x\}$, we have updeg $x=\operatorname{deg} x=m-m^{\prime}$ and, similarly, $m-m^{\prime \prime}=\operatorname{deg} y=\operatorname{updeg} y$. Since $I^{c} \cong J^{c}$, we have

$$
m-m^{\prime}=m-m^{\prime \prime}
$$

Therefore

$$
\operatorname{updeg} x=\operatorname{updeg} y
$$

for every $x, y$ in $H_{1}(P, \leqslant)$.

Consequently, we have the following result from the duality of a CPP.

Corollary 2.3. If $P$ is a $C P P$ and $x, y$ are maximal elements, then

$$
\operatorname{downdeg}(x)=\operatorname{downdeg}(y) \text {. }
$$

Lemma 2.4. Let $P$ be a CPP. Suppose $I$ and $J$ are ideals in $P$ such that $I \cong{ }_{\sigma} J$. Then

$$
\sum_{x \in H_{1}(P, \leqslant) \cap I} \operatorname{updeg} x=\sum_{\sigma(x) \in H_{1}(P, \leqslant) \cap J} \operatorname{updeg} \sigma(x) .
$$

Proof. It is clear by Lemma 2.2 .

Let $I$ be an ideal of $P$ and $T(I)=\{x \in P \mid D \operatorname{Cov}(x)=I\}$. In fact, if $P$ is a poset of height 2 , we have $T(I)$ is a subset of $H_{2}(P, \leqslant)$. The following lemma shows $T(I)$ is not empty if $I$ is an ideal in $H_{1}(P, \leqslant)$.

Lemma 2.5. Let $P$ be a CPP of height 2 and $\operatorname{downdeg}(x)=r$ for all $x \in$ $H_{2}(P, \leqslant)$. Let $I$ be an ideal in $H_{1}(P, \leqslant)$ with $|I|=r$. Then $T(I)$ is not an empty set, i.e., $|T(I)| \geq 1$.

Proof. Since downdeg $(x)=r$ for any $x \in H_{2}(P, \leqslant)$, there is an $r$-element ideal $I_{0}$ in $H_{1}(P, \leqslant)$ such that $I_{0}=\operatorname{DCov}(x)$. On the other hand, assume that there exists an $r$-element ideal $I$ in $H_{1}(P, \leqslant)$ such that $T(I)=\emptyset$. Then $I_{0}^{c}$ and $I^{c}$ are not isomorphic in $P^{d}$, while $I_{0}$ and $I$ are isomorphic in $P$. Hence, this contradicts the hypothesis that $P$ is a CPP. Therefore, $T(I) \neq \emptyset$. 
Proposition 2.6. Let $P$ be a $C P P$ of height 2 and $\operatorname{downdeg}(x)=r$ for all $x \in H_{2}(P, \leqslant)$. Suppose that $I$ and $J$ are ideals in $H_{1}(P, \leqslant)$ with $|I|=|J|=r$. Then $|T(I)|=|T(J)|$.

Proof. Suppose that $I$ and $J$ are ideals in $H_{1}(P, \leqslant)$ with $|I|=|J|=r$. Let $S_{1}=D(I)$ and $S_{2}=D(J)$ in $P^{d}$. Then clearly, $T(I) \subseteq S_{1}$ and $T(J) \subseteq S_{2}$. Since $I$ and $J$ are isomorphic in a CPP $P$, we have $I^{c} \cong J^{c}$ in $P^{d}$. Since $I^{c}$ has no element of $I, I^{c}$ has exactly $|T(I)|$ isolated elements from the definition of $T(I)$. Similarly, $J^{c}$ has exactly $|T(J)|$ isolated elements. Therefore, $|T(I)|=$ $|T(J)|$.

In Proposition 2.6, we emphasize the existence of an invariant $s$ which is the number of elements of $H_{2}(P, \leqslant)$, each of which covers every element of any $r$-element ideal where $r=\operatorname{downdeg}(x)$ for $x \in H_{2}(P, \leqslant)$.

\section{Relationship among four categories of posets}

An ideal of a poset $P$ is clearly a tower of $P$. It is clear that if a poset $P$ is tower-homogeneous, then it is ideal-homogeneous. Therefore, we have the following proposition.

Proposition 3.1. Every tower-homogeneous poset is ideal-homogeneous.

However, the converse is not always true (See Figure 1). As a matter of fact, in Figure 1, the towers $I=\{1,2\}$ and $J=\{3,4\}$ are isomorphic with isomorphism $\alpha$ where $\alpha(1)=3$ and $\alpha(2)=4$. It can be easily seen that $\alpha$ cannot be extended to any automorphism of $P$. Hence, $P$ is not towerhomogeneous. Moreover, $P^{d}$ is not a QCPP as stated previously.

According to Theorem 1.2, a tower-homogeneous poset of height 2 is one of the following cases:

(i) $C(p, q)$ for some $p, q \geq 1$.

(ii) $A(n, k)$ for some $n, k \geq 1$.

(iii) $B(n)$ for $n \geq 3$.

Then, from the definitions of these posets, $C(p, q)$ and $B(n)$ are CPPs, and $A(1, k)$ is a CPP. However, $A(n, k)$ is not a CPP but a QCPP for $n \geq 2$. That is, the converse of Theorem 1.2 is not true.

Theorem 3.2. Every CPP of height 2 is tower-homogeneous.

Proof. For a given CPP $P$ of height 2 and $x \in H_{2}(P, \leqslant)$, let downdeg $(x)$ $=r$ and $\left|H_{1}(P, \leqslant)\right|=m \geq r$. For every $r$-element subset $I$ of $H_{1}(P, \leqslant)$, by Proposition 2.6, there is the fixed number $|T(I)|($ denoted by $t) \geq 1$ of elements of $H_{2}(P, \leqslant)$ which cover all elements of $I$. In order to show the result, we only have to check the following five cases; (1) $r=m$, (2) $r=m-1$ and $t=1$, (3) $r=1$, (4) $1<r<m-1$, and (5) $r=m-1$ and $t>1$.

(1) If $r=m$, then $P$ is isomorphic to $C(r, t)$.

(2) If $r=m-1$ and $t=1$, then $P$ is isomorphic to $B(m)$. 
(3) If $r=1$, then $P$ is isomorphic to $A(m, t)$. If $m \geq 2$, then $A(m, t)$ is not a CPP so that we exclude all other possibilities for $m \geq 2$.

(4) Suppose $1<r<m-1$. Let $x_{0} \in H_{1}(P, \leqslant)$ and $u \in H_{2}(P, \leqslant)$ with $x_{0}<u$. Since $r<m-1$, there exist distinct $y, z \in H_{1}(P, \leqslant)$ incomparable with $u$. Let $S_{1}$ be the set of elements of $H_{1}(P, \leqslant)$ covered by $u$. Since $r>$ 1 , there exists $x_{1} \in S_{1} \backslash\left\{x_{0}\right\}$. Then there exists $v \in H_{2}(P, \leqslant)$ such that $D(v)=\left(S_{1} \backslash\left\{x_{0}\right\}\right) \cup\{y\}$, say $S_{2}$, in $P$. And there exists $w \in H_{2}(P, \leqslant)$ such that $D(w)=\left(S_{1} \backslash\left\{x_{0}, x_{1}\right\}\right) \cup\{y, z\}$, say $S_{3}$, in $P$. Let $I=\{u, v\}$ and $J=\{u, w\}$, then $I \cong J$ in $P^{d}$. We count the decreasing numbers of updeg $(x)$ for $x \in H_{1}(P, \leqslant)$ in $I^{c}$ and $J^{c}$, respectively.

The up-degrees of $x_{0}$ and $y$ in $I^{c}$ are one less than those of $x$ and $y$ in $P$, and the up-degree of $a \in S_{1} \cap S_{2}$ in $I^{c}$ is reduced by two. The up-degrees of others are not changed in $I^{c}$. On the other hand, the up-degrees of $x_{0}, x_{1}, y, z$ in $J^{c}$ are one less than those of $x_{0}, x_{1}, y, z$ in $P$, and the up-degree of $b \in S_{1} \cap S_{3}$ in $J^{c}$ is reduced by two in $J^{c}$. The up-degrees of others are not changed in $J^{c}$. Note that $\left|S_{1} \cap S_{2}\right|=r-1$ and $\left|S_{1} \cap S_{3}\right|=r-2$. That is, in $I^{c}$, there are $r-1$ elements in $H_{1}(P, \leqslant)$ whose up-degrees are reduced by two; however, in $J^{c}$, there are $r-2$ elements in $H_{1}(P, \leqslant)$ whose up-degrees are reduced by two, which means that $I^{c} \nsucceq J^{c}$ in $P$. Therefore, $P$ is not a CPP.

(5) Suppose $r=m-1$ and $t>1$ and $m \geq 3$. Then there exist distinct $x_{0}, y, z \in H_{1}(P, \leqslant)$. Note that $P$ is a CPP. From Lemma 2.2 and its corollary, there exist $u, v, w \in H_{2}(P, \leqslant)$ such that $D(u)=D(v)=H_{1}(P, \leqslant) \backslash\{y\}$ and $D(w)=H_{1}(P, \leqslant) \backslash\{z\}$. Now let $I=\{u, v\}$ and $J=\{u, w\}$ then $I \cong J$ in $P^{d}$. The up-degree of $a \in H_{1}(P, \leqslant)$ is reduced by two in $I^{c}$, and that of $y$ is not changed in $I^{c}$. On the other hand, the up-degree of $b \in D(u) \backslash D(w)$ is reduced by one, and that of $c \in D(u) \cap D(w)$ is reduced by two, and that of $d \in D(w) \backslash$ $D(u)$ is reduced by one. Note that $D(u) \backslash D(w)=\{z\}, D(w) \backslash D(u)=\{y\}$ and $H_{1}(P, \leqslant)=D(u) \cup\{y\}=D(w) \cup\{z\}$. This implies that $|D(u) \cap D(w)|=r-1$. Consequently, in $I^{c}$, there are $r$ elements in $H_{1}(P, \leqslant)$ whose up-degrees are reduced by two; however, in $J^{c}$, there are $r-1$ elements whose up-degrees are reduced by two, which implies that $I^{c} \not J^{c}$ in $P$. Therefore, $P$ is not a CPP.

Through the cases, from (1) to (5), if $P$ is a CPP of height 2 , then $P$ is one of $A(1, t), B(m)$, and $C(m, t)$. Therefore, $P$ is a tower-homogeneous poset.

If a poset $P$ is ideal-homogeneous, then, for ideals $I$ and $J$ with $I \cong{ }_{\sigma} J$, there exists an automorphism $\sigma^{*} \in$ Aut $P$ such that $\left.\sigma^{*}\right|_{I}=\sigma$. The restriction of $\sigma^{*}$ to $I^{c}$ induces an isomorphism from $I^{c}$ to $J^{c}$. Therefore $P$ is a QCPP as stated:

Proposition 3.3. Every ideal-homogeneous poset of height 2 is a QCPP.

For the converse of Proposition 3.3, we give a conjecture as follows.

Conjecture 3.4. Every QCPP of height 2 is ideal-homogeneous.

The results are summarized in Figure 2. 


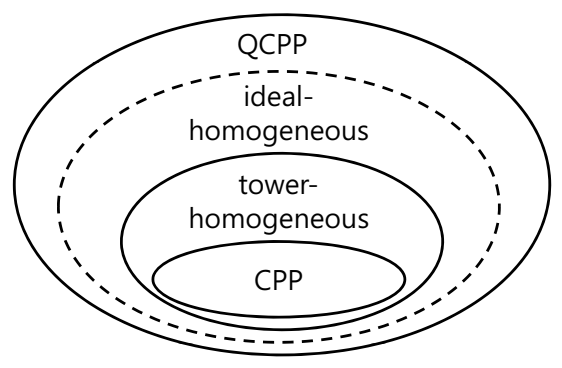

FiguRE 2. A relationship between the homogeneity classes of posets of height 2

Acknowledgment. The authors are grateful to the anonymous referees whose comments improved the clarity of this paper as well as Jong-Yoon Hyun for giving us the idea on a quasi-complement preserved poset and a complement preserved poset.

\section{References}

[1] G. Behrendt, Homogeneity in finite ordered sets, Order 10 (1993), no. 1, 65-75.

[2] B. A. Davey and H. A. Priestley, Introduction to Lattices and Order, Second Edition, Cambridge University Press, 2002.

[3] A. Gardiner, Homogeneity conditions in graphs, J. Combin. Theory Ser. B 24 (1978), no. $3,301-310$.

[4] J. Y. Hyun, in personal communication.

[5] J. H. Schmerl, Countable homogeneous partially ordered sets, Algebra Universalis 9 (1979), no. 3, 317-321.

[6] W. T. Trotter, Combinatorics and Partially Ordered sets: Dimension theory, The Johns Hopkins University Press, 1992.

Gab-Byung Chae

Division of Mathematics and Informational Statistics

Institute of Natural Basic Sciences

WONKWANG UNIVERSITY

IKSAN 570-749, KOREA

E-mail address: rivendell@wku.ac.kr

Minseok Cheong

Department of Mathematics

SOGANG UNIVERSITY

Seoul 121-742, Korea

E-mail address: macross@sogang.ac.kr

SANG-MOK KIM

Department of Mathematics

KWANGWOON UNIVERSITY

SEOUl 139-701, Korea

E-mail address: smkim@kw.ac.kr 